\title{
Chapter 12 \\ Optimization-Based Control of \\ Microgrids for Ancillary Services \\ Provision and Islanded Operation
}

\author{
Alessio La Bella
}

\subsection{Introduction}

Climate change is today a well-known issue. The average world temperature is continuously rising, due to the high concentration of greenhouse gasses, and the negative effects on the environment are already more than evident. Among the involved causes, electricity production is one of the largest responsible, accounting for around one third of the global greenhouse gas emissions every year. Therefore, the actual centralized generation system, where most of the power is provided by fossil-based power plants, is no more a sustainable paradigm and this is today required to shift to a more distributed architecture, relying on a conspicuous spread of Renewable Energy Sources (RESs), as confirmed by the European Union in the Directive 2018/2001. The energy transition is not a straightforward path and many technical challenges must be tackled. A main issue is that renewable generation is intermittent and nondeterministic since it often depends on weather conditions, e.g. for wind-turbine and photovoltaic generators. This implies that RESs, by themselves, cannot guarantee the continuous balance between power generation and demand, which is a fundamental requirement not only to guarantee the secure power supply but also for the frequency' and voltages' stability [1]. A solution to overcome this issue is to combine the RESs' diffusion with a parallel integration of other distributed dispatchable resources, such as battery systems and smart modulable loads, which can be properly controlled to adapt their power patterns ensuring the power balance in the electrical system, i.e. providing the so-called ancillary services [2]. Nevertheless, this decentralized paradigm involves increasing coordination issues with respect to the traditional system, requiring the efficient management of the widespread distributed resources based on the real-time needs of the electrical system.

\footnotetext{
A. La Bella (凶)

Politecnico di Milano, Piazza Leonardo da Vinci 32, 20133 Milan, Italy

e-mail: alessio.labella@polimi.it

(C) The Author(s) 2021

A. Geraci (ed.), Special Topics in Information Technology,

PoliMI SpringerBriefs, https://doi.org/10.1007/978-3-030-62476-7_12
} 


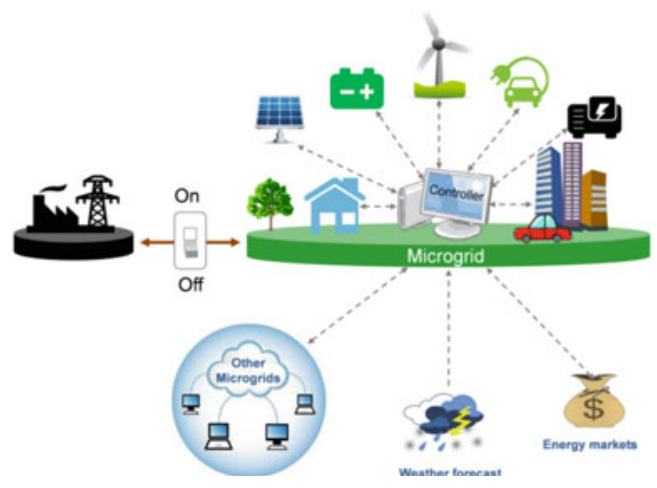

Fig. 12.1 Schematic of a generic MG [https://building-microgrid.lbl.gov]

In this context, MicroGrids (MGs) have been devised as a great solution to enhance the flexibility and controllability of the future electrical system. A MG is small-scale grid, usually large as a city district, incorporating RESs, batteries, micro-generators, and smart loads, which are then regulated by proper control architectures. The deployment of MGs would bring several benefits to the electrical system, as they allow a more effective management of RESs, thanks to the co-location with other dispatchable devices that can balance their variability [3]. Another significant advantage derives by the flexible nature of MGs, as they can be operated either grid-connected or in islanded mode. A schematic of a MG is shown in Fig. 12.1.

Nevertheless, MGs are still not recognized as a competitive alternative to fossilbased power plants. In fact, the electrical regulations on ancillary services provision are characterized by too high power requirements for single microgrids, as they have been traditionally requested to big power plants [4]. On the other hand, the MG islanded mode still involves several technical challenges due to the absence of the main utility's support. In fact, local resources must be controlled both to efficiently ensure the MG internal power balance, despite the presence of uncertain RESs, and to internally stabilize the MG frequency and voltages [5].

This doctoral thesis therefore have aimed to design several control architectures and algorithms to properly and efficiently operate MGs in grid-connected and islanded mode, overcoming the mentioned issues and so fostering the MGs' integration to facilitate the energy transition [6]. This summary brief is structured as follows. Section 12.2 provides an overview of different optimization-based control strategies for MGs to become valuable providers of ancillary services. Then, the designed control systems for islanded MGs are described in Sect. 12.3. Section 12.4 concludes this brief with some final remarks, describing possible future research directions.

\subsection{Microgrids Aggregators Providing Ancillary Services}

As mentioned in Sect. 12.1, MGs are usually characterized by a limited power capability, having to satisfy also their internal loads, and so they are not significant players 
for the ancillary service provision. To solve this issue, this doctoral thesis proposes several control architectures to jointly coordinate an aggregator of multiple interconnected MGs, properly regulated by an Aggregator Supervisor (AGS), so that they can act as a unique significant entity for the system operators, potentially capable of providing ancillary services [7]. Aggregation is in fact an effective solution to foster the participation of small-scale distributed resources to the ancillary services provision, as also recognized by the European Union in the Regulation 2017/2195 [8]. To properly accomplish this task, aggregators must perform the following operations:

- During the offline operation, e.g. the day-ahead, the AGS must define the optimal overall power exchange of the MGs Aggregator (MG-AG) with the main utility, considering the energy prices and the operation costs of the MGs' units. This phase must also consider the allocation of a proper amount of power reserve in the MG-AG, respecting the minimum requirements imposed by the regulations.

- During the online operation, e.g. in the intra-day, the AGS must be able to promptly reschedule MGs operations to compensate power imbalances in the electrical system, varying the pre-scheduled MG-AG output power as requested by the system operators.

- During the real-time operation, the AGS must ensure that the agreed MG-AG power profile is maintained despite the presence of non-deterministic RESs and loads inside the MG-AG, therefore all the internal unexpected imbalances must be promptly compensated.

These three phases will be the core of the control algorithms described in the next three paragraphs, respectively. It should be underlined that controlling a MG-AG is a large-scale and computationally-intensive problem and moreover, it is necessary to preserve MGs' internal information and the local control of their units, being them private facilities. Because of this, the proposed control algorithms are not defined according to pure centralized approaches, but novel distributed and hierarchical schemes are proposed with enhanced optimality and scalability properties, so that their performances are independent on the size of the aggregation.

\subsubsection{Offline Economic Dispatch and Power Reserve Procurement}

This offline phase aims to define the MG-AG optimal output power considering the energy prices and the internal MGs costs, as well as to procure a required minimum amount of power reserve. Additionally, MGs operations must be coordinated also to avoid over-voltage and congestion issues in the electrical network, which can be easily occur in presence of distributed generation.

To accomplish these tasks, firstly the optimal MG-AG power profiles are scheduled, considering economic objectives and the ancillary service provision. Then, in a second step, the electrical feasibility of the scheduled profiles is checked and modifications are implemented if necessary. For the first task, it should be considered that 
each MG wants to optimize the use of its own units, considering local cost functions and constraints. However, MGs optimization problems are all linked by coupling constraints related both to the MG-AG output power to sell/buy to/from the main utility and to the MG-AG power reserve that must be allocated. This implies that pure decentralized approaches can not be implemented as an interaction between the different MGs is needed. Because of this, this first step is addressed through the definition of a distributed optimization algorithm based on the dual decomposition theory, precisely the Alternating Direction Method of Multipliers (ADMM) [9]. Avoiding all the mathematical details of this approach, this technique allows to remove the constraints coupling the MGs optimization problems, expressing their violations as properly weighted costs through the so-called Lagrangian relaxation [10]. This makes the overall optimization problem separable, and so it can be distributed among the MGs and the AGS. The separable optimization problems are then solved through an iterative negotiation between the AGS and the MGs, which at convergence achieves the optimal solution and the feasibility of the coupling constraints. Precisely, the following sequential operations are iteratively executed:

- The AGS sends to the MGs properly defined internal prices, denoted as dual variables, for the MGs power output and allocated power reserve.

- MGs solve in parallel their optimization problems considering the AGS internal prices and their own units' costs. Then, they communicate to the AGS their optimal output power and allocated reserve.

- The AGS gathers this information and solves an internal optimization problem. Based on its optimal solution, it properly updates the dual variables trying to drive MGs towards the optimal and feasible solution.

The main advantage of the proposed approach is that MGs do not have to communicate their internal information to the AGS, as they directly optimize their internal units, and moreover MGs operate in parallel, implying that the overall computational time does not rise with their number. If some mild assumptions are respected (e.g. convexity of the optimization problems), the described algorithm converges to the same optimal objective of the centralized system, i.e. the case where the AGS has full knowledge and control of the MGs internal units. Having defined the optimal power profiles of the MG-AG, an additional optimization procedure is then performed to check power flow feasibility, considering the limitations on nodal voltages and line currents. This task is not easily solvable through a distributed approach since the corresponding optimization problem is inevitably non-convex, due to the power flow equations, and therefore convergence and feasibility issues arise [11]. Therefore, MGs are properly modeled as equivalent generators, which allows to address the electrical feasibility in a pure centralized way, without however requiring MGs to communicate their internal information and involving computational issues.

Figure 12.2 shows some results of the described procedure, considering as benchmark an aggregation of 4 MGs connected to the IEEE 37 bus system [12]. In particular, from Fig. 12.2a the optimal output power computed by the distributed algorithm is compared to the optimal solution of the centralized system, where it is evident that the same solution is achieved. Figure $12.2 \mathrm{~b}$ shows instead the difference of the 


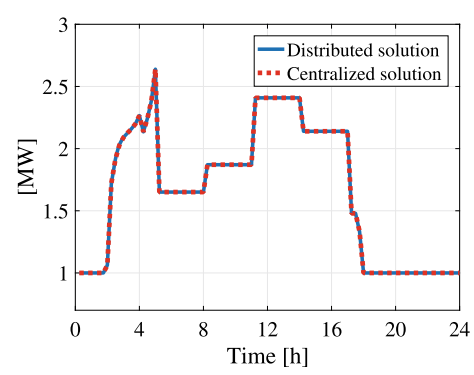

(a)



(b)

Fig. 12.2 a MG-AG optimal power output; b Optimality gap between centralized and distributed solution over the iterations of the proposed algorithm

optimal cost function between the centralized and the distributed solution over the number of iterations, considering also a varying number of MGs inside the MG-AG. As notable, the optimality gap always converges to zero in around 70 iterations, independently on the number of MGs, given the scalability properties of the proposed approach.

\subsubsection{Online External Provision of Ancillary Services}

During the online operation, the AGS has to reschedule the MG-AG operations as requested for the external provision of ancillary services. Precisely, the system operators can request the AGS to increase, or decrease, the MG-AG output power over a specific time period, defined as request period. Moreover, the AGS can also periodically offer additional power reserve to the system operators, if available.

The AGS has to respect additional constraints while performing these tasks. In fact, the MG-AG output power profile must be varied just during the request period, and not in the time instants subsequent it, avoiding the so-called rebound effect [13]. Moreover, neither the pre-agreed power reserve must be affected in the time instants subsequent to the request period, since ancillary services may be later requested.

It should be noted that MGs can decide to interrupt, shift or modulate some controllable loads to satisfy the system operators' requests. This results in the introduction of mixed-integer models, implying that standard distributed approaches may lead to sub-optimality and feasibility issues [14]. Therefore a novel hierarchical technique is proposed to perform this task, based on the definition of the flexibility function concept. These functions express the maximum and minimum power variation that each MG can provide over the request period, as well as the additional cost that each MG afford for any requested power variation. These become effective tools for the AGS to quickly, and optimally, reschedule the MG-AG operations as requested, and to offer additional power reserve if available. Moreover, also in this case MGs do not have to communicate their internal models and characteristics, but just this 
flexibility information. It is not simple to analytically characterize the MG flexibility functions, as they are inherently non-linear and non-convex due to the mixed-integer MGs modelling. However, it is shown in the doctoral thesis how each MG can easily compute a convex approximation of its flexibility function. This allows the AGS to be extremely efficient in computing the optimal solution, as it solves a convexification of the MG-AG rescheduling problem. The test results of this approach revealed to be particularly promising. Despite the introduced approximations, the optimality gap between the centralized solution and the solution computed by the proposed approach reached at maximum $0.1 \%$. Moreover, the proposed technique revealed to be much faster than the centralized case in computing the MG-AG rescheduling, reaching a reduction of the $80 \%$ for the overall computational time.

\subsubsection{Real-Time Self-balancing of Internal Power Uncertainties}

The last operation concerns the tracking of the agreed MG-AG power profile, which is a critical task due to the presence of several non-controllable and non-deterministic RESs and loads inside the MG-AG, as their output power often deviate with respect to the forecasts. To overcome this issue, it is proposed to coordinate MGs to exploit the remaining power reserve, i.e. the one not externally requested by the system operators, compensating the internal power variability of the MG-AG. To perform this task, a scalable and prompt control architecture is required, as power imbalances must be quickly balanced even in large-scale electrical networks. This implies that neither centralized nor pure distributed approaches are advisable, as the former are not scalable, and the latter are not prompt but usually involve iterative procedures. Therefore, a novel control approach is here designed, which schematic is depicted in Fig. 12.3.

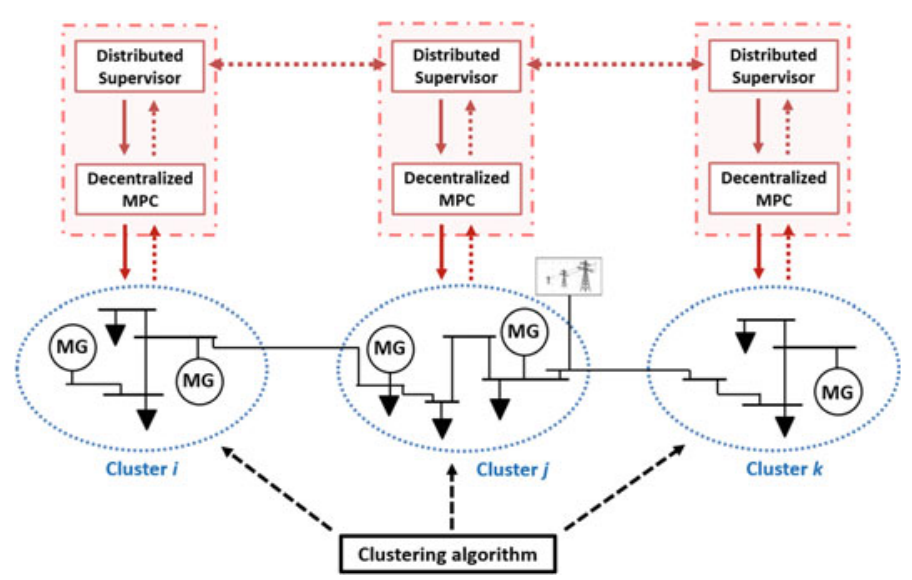

Fig. 12.3 Schematic of the proposed control architecture for real-time power balancing 


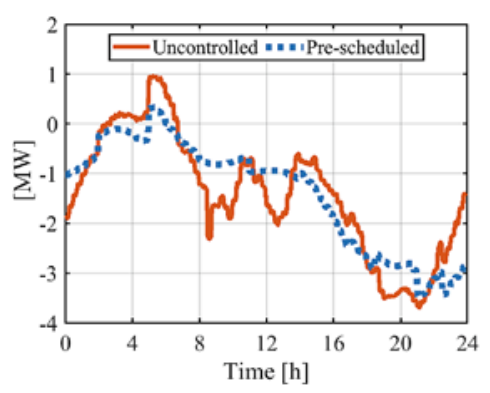

(a)

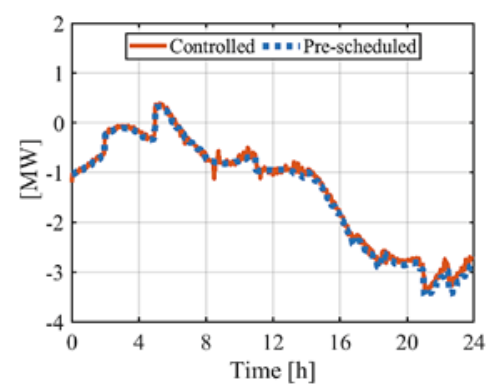

(b)

Fig. 12.4 MG-AG power exchange in the uncontrolled (a) and controlled case (b)

The first step of the approach is based on a properly designed clustering procedure, with the objective of creating network clusters that are as self-sufficient as possible in compensating unexpected internal power imbalances. The proposed clustering algorithm takes into account the remaining power reserves in MGs and the worst-case deviations of loads and RESs. Then, a two-layer control architecture is implemented. The lower layer is constituted by a decentralized framework of Model Predictive Control (MPC) regulators, each one managing a network cluster by coordinating the MGs to balance the cluster' power variability. However, if large power deviations occur in a cluster and the remaining power reserve is not sufficient to compensate it, the decentralized MPC regulator can issue a power request to the upper layer: the Distributed Supervisor. This is a fully distributed control scheme which defines the optimal power exchanges among the different clusters so that the overall network remains always balanced. This scheme is based on the Distributed Consensus ADMM algorithm, which allows the direct interaction between the different supervising agents [15].

The proposed architecture has been tested on several IEEE benchmarks, such as the 37,118 and the 123 bus-systems. Figure 12.4 reports some results for the IEEE 37 bus-system, comparing the MG-AG power exchange with the main utility if the proposed control architecture is applied and if it is not. It is evident that the variability of loads and RESs seriously affects the MG-AG output power, as it significantly deviates from the pre-scheduled profile if imbalances are not promptly restored. On the other hand, the proposed scheme allows almost a perfect tracking of the pre-scheduled power profile, through the efficient exploitation of local MGs. It is worth noticing that the upper supervising layer, although distributed, is particularly fast in computing the optimal solution as most of the model complexity is addressed by the local MPC regulators. It is also worth noticing that the two control layers are decoupled, meaning that the MPC regulators continuously and autonomously operate to compensate all unexpected power deviations, while the supervisor is activated to optimally redefine the power exchanges among clusters just when necessary. 


\subsection{Hierarchical Model Predictive Control Architectures for Islanded Microgrids}

A MG can be also operated in islanded mode, just relying on its own local sources. This is a valid solution to ensure power supply in critical facilities in case of blackout events (e.g. hospitals), and to electrify rural areas in a sustainable way, as in some developing countries where a consistent electric infrastructure is still absent. However, as mentioned, the islanded mode requires efficient and prompt architectures to control local resources, ensuring both the optimal MG management and the stabilization of the internal frequency and voltages. Since these tasks involve different time scales and system modelling, multi-layer control architectures are proposed considering MGs with alternating current networks (AC-MGs) and also MGs with direct current networks (DC-MGs), which have recently raised a significant interest for their high efficiency [16]. Among the proposed solutions, the three-layer control architecture for DC-MGs, designed during a visiting period at the Automatic Control Laboratory of EPFL, is here described. In case the reader is interested to the designed control architectures for islanded AC-MGs, please refers to [6, 17, 18].

The schematic of the proposed control architecture for islanded DC-MGs is depicted in Fig. 12.5. At the upper layer, a hybrid-MPC system is implemented, executed with a sampling time of $15 \mathrm{~min}$, able to consider units' constraints, weather predictions, mixed-integer models and several economic aspects. The objective of this control layer is to ensure the optimal MG power balance, defining the generators' power references and switching on, or off, some MGs units when convenient. Then, low-level plug-and-play fast voltage controllers are designed, acting at the converter interfaces of the generation units, having the particular characteristic of guaranteeing the voltage stability also if generation units are switched on, or off. To properly

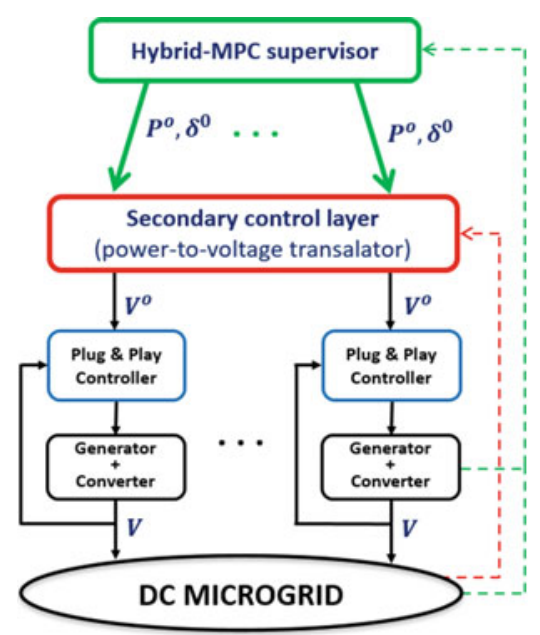

Fig. 12.5 Three-layer control architecture for an islanded DC-MG 
interface these two layers, an innovative secondary optimization-based system is also designed, properly translating the power references provided by the hybrid MPC to the voltage references needed by the plug-and-play controllers. The theoretical properties of this interfacing layer have been analysed, due to its nonlinear and non-convex nature, and it has been proved that, under realistic and easy-verifiable assumptions, a solution for the power-to-voltage translation always exists and, moreover, this is unique [19]. These properties revealed to be particularly significant for ensuring the proper and secure operation of the whole architecture. The hierarchical control system has been tested on real DC-MG benchmark, showing its significant performances in ensuring the optimal and stable operation in many different conditions.

\subsection{Conclusions}

This chapter aimed to give an overview of the different control architectures designed for microgrids, considered the key-solution for enhancing the spread of renewable energy sources in the electrical system [6]. In particular, the control strategies presented in Sect. 12.2 showed to be really effective solutions to make microgrids fundamental players in the electrical system, as they can cooperate as part of a unique aggregator to efficiently provide ancillary services. Moreover, the hierarchical control architectures designed for islanded microgrids, briefly described in Sect. 12.3, allow the optimal energy management of the local sources and to stabilize the internal frequency and voltages also in case the main grid support is missing. Future research directions may involve the use on historical data of renewable sources' production and loads' consumption for improving the control design and increasing the energy efficiency. Additional research effort may be also devoted to the design of multiagent algorithms for generic problem structures, as microgrids may easily involve non-linear models, constraints and costs and this should not affect their efficient coordination in supporting the upcoming energy transition.

\section{References}

1. Kundur P, Balu NJ, Lauby MG (1994) Power system stability and control. McGraw-Hill, New York

2. Joos G, Ooi BT, McGillis D, Galiana FD, Marceau R (2000) The potential of distributed generation to provide ancillary services. In: 2000 IEEE power engineering society summer meeting, vol 3, pp 1762-1767

3. Hatziargyriou ND, Anastasiadis AG, Vasiljevska J, Tsikalakis AG (2009) Quantification of economic, environmental and operational benefits of microgrids. In: 2009 IEEE Bucharest PowerTech, pp 1-8

4. Yuen C, Oudalov A (2007) The feasibility and profitability of ancillary services provision from multi-microgrids. In: 2007 IEEE Lausanne Power Tech. IEEE, pp 598-603

5. Lasseter RH, Paigi P (2004) Microgrid: a conceptual solution. In: 2004 IEEE 35 th annual power electronics specialists conference (IEEE Cat. No. 04CH37551), vol 6. IEEE, pp 4285-4290 
6. La Bella A (2020) Optimization-based control of microgrids for ancillary services provision and islanded operation. Doctoral dissertation, Italy

7. La Bella A, Farina M, Sandroni C, Scattolini R (2018) Microgrids aggregation management providing ancillary services. In: 2018 European control conference (ECC). IEEE, pp 11361141

8. Poplavskaya K, De Vries L (2019) Distributed energy resources and the organized balancing market: a symbiosis yet? Case of three European balancing markets. Energy Policy 126:264276

9. Boyd S, Parikh N, Chu E (2011) Distributed optimization and statistical learning via the alternating direction method of multipliers. Now Publishers Inc

10. Bertsekas DP (1997) Nonlinear programming. J Oper Res Soc 48(3):334-334

11. Molzahn DK, Dörfler F, Sandberg H, Low SH, Chakrabarti S, Baldick R, Lavaei J (2017) A survey of distributed optimization and control algorithms for electric power systems. IEEE Trans Smart Grid 8(6):2941-2962

12. La Bella A, Farina M, Sandroni C, Scattolini R (Nov, 2020) Design of aggregators for the dayahead management of microgrids providing active and reactive power services. IEEE Trans Control Syst Technol 28(6):2616-2624, https://doi.org/10.1109/TCST.2019.2939992

13. Samad T, Koch E, Stluka P (2016) Automated demand response for smart buildings and microgrids: the state of the practice and research challenges. Proc IEEE 104(4):726-744

14. Kim SJ, Giannakis GB (2013) Scalable and robust demand response with mixed-integer constraints. IEEE Trans Smart Grid 4(4):2089-2099

15. Chang TH (2016) A proximal dual consensus ADMM method for multi-agent constrained optimization. IEEE Trans Signal Process 64(14):3719-3734

16. Justo JJ, Mwasilu F, Lee J, Jung JW (2013) AC-microgrids versus DC-microgrids with distributed energy resources: a review. Renew Sustain Energy Rev 24:387-405

17. La Bella A, Cominesi SR, Sandroni C, Scattolini R (2016) Hierarchical predictive control of microgrids in islanded operation. IEEE Trans Autom Sci Eng 14(2):536-546

18. La Bella A, Negri S, Scattolini R, Tironi E (2018) A two-layer control architecture for islanded AC microgrids with storage devices. In: 2018 IEEE conference on control technology and applications (CCTA), pp 1421-1426

19. La Bella A, Nahata P, Ferrari-Trecate G (2019) A supervisory control structure for voltagecontrolled islanded DC microgrids. In: 2019 IEEE 58th conference on decision and control (CDC). IEEE, pp 6566-6571

Open Access This chapter is licensed under the terms of the Creative Commons Attribution 4.0 International License (http://creativecommons.org/licenses/by/4.0/), which permits use, sharing, adaptation, distribution and reproduction in any medium or format, as long as you give appropriate credit to the original author(s) and the source, provide a link to the Creative Commons license and indicate if changes were made.

The images or other third party material in this chapter are included in the chapter's Creative Commons license, unless indicated otherwise in a credit line to the material. If material is not included in the chapter's Creative Commons license and your intended use is not permitted by statutory regulation or exceeds the permitted use, you will need to obtain permission directly from the copyright holder. 